\title{
Prediction of paired-associate learning from a complex indicator of associative strength
}

\author{
MARIGOLD LINTON and MARSHA JEWETT* \\ San Diego State College, San Diego, Calif. 92115 \\ and \\ S. JOYCE BROTSKY \\ San Fernando Valley State College, Northridge, Calif. 91324
}

Forty-eight Ss learned successively four homogeneous lists constructed by pairing stimuli with their most frequent, their second, their third, or their fourth normative response. Predictions of paired-associate learning from associative strength defined by both rank and normative frequency of response were confirmed. The results were attributed to (1) utilization of a complex indicator of associative strength, and (2) establishment of conditions which facilitated development of appropriate response sets.

Speed of paired-associate learning (PAL) should be predictable from the normative free-association strength (FAS) between word pairs for at least two reasons: (1) Strongly learned habits which are reflected as common responses in the word-association test (WAT) should be elicited by stimuli in a PAL task, and (2) response sets which may mediate responses in a WAT should be producible in a PAL task either through instructions or through manipulation of the S-R pairs employed. When children are used as Ss, speed of PAL is a function of associative strength as indexed by absolute normative frequencies (Wicklund, Palermo, \& Jenkins, 1964). With adult Ss, however, the relationship has not been consistently found. One explanation of the similar performance for adults on all pairs is that all pairs are learned through the priming of habits whose strength are near asymptote.

An alternate explanation is that normative frequency derived from WAT may not provide an adequate index of associative strength for adult Ss. A series of studies has attempted to improve prediction of PAL through use of alternative measures of associative strength (e.g., Kammann, 1968) or through additional specification of stimulus characteristics (e.g., Martin, 1964). In some cases, it seems apparent that absolute frequency of a response may not provide an adequate basis for defining associative strength. This is exemplified by the

*Now at the University of California, Los Angeles. This research was performed in part while the second author was a National Science Foundation Undergraduate Research Participant. This research also received support, in part, from a faculty research award to the first author from the San Diego State College Foundation and from a research grant to the third author from the San Fernando Valley State College Foundation. following cases (Bousfield, Cohen. Whitmarsh, \& Kincaid, 1961): NAKED occurs as a response to BARE for $11 \%$ of the people, CAT to ANIMAL for $11 \%$, and PIE to APPLE for $11 \%$. If associative strength were indexed solely by absolute normative frequency, no differences in ease of learning the three word pairs would be predicted. Prediction may be improved by considering that NAKED is the primary response (R1) to BARE, CAT is the secondary response (R2) to ANIMAL whose strong $\mathrm{R} 1$ is DOG, and PIE is the R4 to APPLE, with three competing responses of higher rank. It seemed probable that consideration of both relative frequency (rank) and absolute frequency would produce better estimates of associative strength and more homogeneous lists than either variable used alone. Greater list homogeneity may influence the learnability of a paired-associate list by changing the probability that Ss will adopt an appropriate response set.

\section{STIMULUS MATERIALS AND DESIGN}

Two groups of stimulus words were selected from the Connecticut Free Associational Norms (Bousfield et al, 1961). For Group 1, the associative frequencies of R1 and R4 were specified with the frequency of R2 and R3 not controlled; for Group 2, the frequencies of R2 and R3 were specified with the frequency of R1 and R4 not controlled. Group 1 consisted of stimuli whose mean FAS for R1 was $77 \%$ (range $=67 \%-91 \%$ ) and for R4 was $2 \%$ (range $=0.7 \%-3 \%$ ). Group 2 consisted of stimuli whose mean FAS for R2 and R3 was $23 \%$ (range $=20 \%-32 \%$ ) and $8 \%$ (range $=3 \%-16 \%)$, respectively. The remaining frequencies were not controlled. Group 1 and Group 2 identified two very disparate sets of stimulus words. Group 1 stimuli had a very strong primary, but the mean FAS of responses from R1 to R4 dropped rapidly $(77 \%, 6 \%, 3 \%$, and $2 \%$, respectively). Group 2 stimuli had a weaker primary and the mean FAS of lower rank responses dropped off more slowly from R1 to R4 (35\%, 25\%, 8\%, and 5\%, respectively).

Two sets of 10 stimuli drawn from Group 1 comprised List 1 and List 4 . List 1 consisted of S-R1 pairings and List 4 of S-R4 pairings. Two sets of 10 stimuli similarly drawn from Group 2 comprised List 2 (S-R2) and List 3 (S-R3). No word occurred in more than one list. The mean FAS in Replication A for List 1 through List 4 , respectively. were $76 \%, 25 \%, 10 \%$, and $2 \%$. To rulc out effects due to stimulus word, per se. four new lists were developed for Replication B as follows: Stimuli in List 1 were paired with their appropriate R4, and stimuli in List 4 were paired with their appropriate $R 1$. Thus, stimuli in List 1 became the stimuli for List 4 in Replication $B$. The equivalent reversal was performed for Lists 2 and 3. The mean FAS in Replication B for Lists 1-4. respectively, were $79 \%, 22 \%, 7 \%$, and $2 \%$. Within each list, five of the stimuli were Thorndike-Lorge (1944) AA words and the other five had a language frequency (LF) of less than 50 /million. Within the restricted degrees of freedom remaining, LF values of responses were roughly equated. The number of $\mathrm{A}$ or $\mathrm{AA}$ responses in each list ranged from seven to nine while the $L F$ of the remaining responses was less than $50 /$ million.

\section{SUBJECTS AND PROCEDURE}

The Ss were 24 male and 24 female psychology students at San Diego State College. Each $S$ learned successively the four lists of pairs from either Replication A or Replication B in a standard anticipation paired-associate procedure. Each $S$ learned all pairs at either a 1.5 - or a 2.0 -sec rate. Stimuli were presented via Stowe Memory Drum. Order and sequence of the lists were counterbalanced. There were three random orders of each list. Criterion of learning was two successive errorless trials.

\section{RESULTS}

Associative strength (list) and the ordinal position in which a list was learned were within-Ss variables, while replication and presentation rate were between-Ss variables. An analysis of variance 2 by 2 by 4 by 4 crossover design was applied to $\log (X-1)$ number of trials to criterion. The transformation was performed since the rapid learning of List 1 would otherwise have produced a violation of the assumption of homogeneity of variance.

The mean number of trials to criterion were $2.23,3.14,3.79$, and 4.40 for List 1 through List 4 , respectively. These mean 
values omit the study trial but include the two trials to criterion. These differences due to list were significant, $F=42.08$, $\mathrm{df}=3 / 93, \quad p<.05$. Duncan's range test indicated that all these means were significantly different from each other. In addition, List by Replication interaction was significant, $F=4.87, \quad \mathrm{df}=3 / 93$, $p<.05$. None of the other effects was significant.

The difference between the mean overall number of trials to criterion for the four lists combined for women (16.08) and for men (19.04) was significant, $t=3.11$, $\mathrm{df}=46, \mathrm{p}<.05$. A $\mathrm{t}$ test was performed on number of errors for low- vs high-frequency stimulus word pairs within each list for the two replications combined. The differences were not significant for any list.

\section{DISCUSSION}

The present study clearly confirms a general relationship between PAL and FAS. The range of values of absolute normative frequency was considerable-from $2 \%$ to $77 \%$. Ranks varied only from 1 to 4 . The relationship was found using conventional PAL procedures, with each of two presentation rates, and for both men and women. Further, each list contained words of high and low LF and there were no significant differences in PAL for high and low LF stimuli. The present findings do not confirm those of Postman (1962) who found faster learning of strong than of weak associates only with low LF stimuli, nor are they consistent with those of Martin (1964) who found no differences between strong and medium FAS pairs regardless of LF. Why has the present study found a simple relationship between FAS and PAL when other researchers have failed to do so? Two procedures may be responsible for these differences. The present study utilized a complex indicator (CI) of associative strength: (1) the relative frequency of the response word, and (2) the absolute frequency of the response word as well as that of an additional response in the hierarchy. The use of a CI to define FAS seems to have merit. The choice of the components of CIs and their relative weighting must remain an empirical matter.

In the present study the following specific rankings had been predicted (from best to poorest learning): S-R1 > S-R2 > S-R3 > S-R4. Complex rules may sometimes be required to determine associative strength from combinations of the FAS indicators. In the present case, predictions for S-R1 pairs were clearcut. Pairs with the strong FAS, high rank in the hierarchy, and few strong competitors should be readily learned. The predictions for S-R2, too, were relatively simple. These pairs had relatively strong FAS, were high in normative rank, and had only one stronger competitor. Determining the relative strength of S-R3 and S-R4 in the present context was more difficult. Was the associative strength of a S-R3 pair with a response of relatively low rank, low FAS, with two moderate (a total of $40 \%$ ) more frequent competitors stronger than that of a S-R4 pair, where the response was of still lower rank and FAS, but with one very strong and two relatively weak (a total of $85 \%$ ) more frequent competitors? The total more-dominant FAS competition, disregarding the pattern, suggested the superiority of S-R3 to S-R4. This ordering of associative strength, although confirmed in the present study, is inconsistent with the findings of Shapiro (1968), who found shorter latencies in the learning of nondominant responses with strong dominant competitors.

The present study utilized homogeneous PAL lists, since it was reasoned that such lists would facilitate a response set similar to that in the WAT. Such a set would make associations available during WAT highly probable and should facilitate learning of all S-R pairs. It seems likely that Ss would have more available the high-frequency/high-rank responses than the low-frequency/low-rank responses because it is easier to isolate the "correct" word when it is a highly dominant primary with few competing responses. Presumably when the to-be-learned response is one of

several words that could "go with" the stimulus and not necessarily the one highest in S's own response hierarchy, additional rules specific to individual pairs may be required, a process which might require additional time. The failure to find a significant interaction between FAS and presentation time suggests that if response set is a factor, it operates similarly for all levels of associative strength.

\title{
REFERENCES
}

BOUSFIELD, W. A., COHEN, B. H., WHITMARSH, G. A., \& KINCAID, W. D. The Connecticut free associational norms. Technical Report No.35, November 1961, University of Connecticut, Contract Nonr-631(00), Office of Naval Research.

KAMMANN, R. Associability: A study of the properties of associative ratings and the role of association in word-word learning. Journal of Experimental Psychology Monograph, 1968, 78 (4, Whole Part 2).

MARTIN, J. G. Associative strength and word frequency in paired-associate learning. Journal of Verbal Learning \& Verbal Behavior, 1964, $3,317-320$.

POSTMAN, L. The effects of language habits on the acquisition and retention of verbal associations. Journal of Experimental Psychology, 1962, 64, 7-19.

SHAPIRO, S. I. Paired-associate response latencies as a function of free association strength. Journal of Experimental Psychology, $1968,77,223-231$.

THORNDIKE, E. L., \& LORGE, 1. The teacher's wordbook of 30,000 words. New York: Bureau of Publications, Teachers College, Columbia University, 1944.

WICKLUND, D. A., PALERMO, D. S., \& JENKINS, J. J. The effects of associative strength and response hierarchy on paired-associate learning. Journal of Verbal Learning \& Verbal Behavior, 1964, 3, 413-420.

\section{Rigidity and instructions in relation to two-flash fusion measures}

\author{
J. E. TONG, University of Guelph, Guelph, Ontario, Canada \\ and \\ D. GAIL GROUND, University of Western Ontario, London, Canada
}

It has been suggested that scores from constant-stimuli procedures with signal-detection analyses may be influenced by a personality variable termed "rigidity." To test this in connection with the discrimination of temporally paired flashes, high-rigidity and low-rigidity Ss were given both facilitating and inhibiting instructions with the two-flash fusion task. Signal-detection analyses indicated significantly higher threshold measures under both conditions for low-ridigity Ss. Inhibiting instructions raised both threshold and criterion scores for both groups, the low-rigidity group showing the greater criterion shift.

Recent papers have indicated the feasibility of signal-detection procedures for the study of two-flash fusion threshold (TFF) (Dorosh et al, 1970), the significance of signal-detection measures for the investigation of drug effects

\footnotetext{
*This research was supported by a grant from the National Research Council of Canada to J. E. Tong.
}

(Gruzelier \& Corballis, 1970), and induced autonomic change (Boissonneault et al, 1970). Treisman \& Watts (1966) developed a signal-detection model, based on a method of constant-stimuli presentation format, which offers certain practical advantages for TFF work. The Treisman and Watts procedure enables the isolation of three scores, namely, threshold, criterion, and sensitivity (D'Amato, 1970, 